\title{
PRODUÇÃO DE CHAPAS DE PARTÍCULAS DE MADEIRA DE DUAS ESPÉCIES NATIVAS DA MATA ATLÂNTICA E SUAS COMBINAÇÕES
}

\section{PARTICLEBOARD MADE FROM TWO NATIVE SPECIES OF THE MATA ATLÂNTICA AND IT'S COMBINATIONS}

\author{
Graziela Baptista Vidaurre ${ }^{1}$ Avelino Nogueira da Silva ${ }^{1} \quad$ José D. de Sá Rocha $^{1}$ \\ Edvá Oliveira Brito ${ }^{2}$
}

\section{RESUMO}

O objetivo deste trabalho foi produzir chapas de partículas "Strand" e maravalhas partindo de Schizolobium parahyba e Erytrina verna e suas combinações. Os parâmetros de manufatura foram: densidade nominal de $0,65 \mathrm{~g} / \mathrm{cm}^{3}$, temperatura de prensagem de $180^{\circ} \mathrm{C}$, tempo de prensagem de 14 minutos, resina fenolformaldeído a $8 \%$ e umidade das partículas a 5\%. Os melhores resultados para o ensaio de flexão estática foram encontrados quando se utilizou guapuruvu puro e/ou na capa, embora a maioria dos tratamentos tenha apresentado valores superiores aos estabelecidos pela norma canadense (CSA O437-0, 1993). Em relação à ligação interna, $50 \%$ dos tratamentos apresentaram valores superiores aos estabelecidos pela norma canadense. Os valores de absorção de água e inchamento em espessura foram considerados altos para todos os tratamentos, devendo-se considerar que não foi utilizado parafina para a confecção das chapas.

Palavras-chave: chapas de partículas; Oriented Strand Board; Schizolobium parahyb; Erytrina verna.

\section{ABSTRACT}

The aim of this work was to manufacture strand and shaving particleboards from Schizolobium parahyba and Erytrina verna and their combinations. The parameters of manufacture were: nominal density $0,65 \mathrm{~g} / \mathrm{cm}^{3}$, press temperature $180{ }^{\circ} \mathrm{C}$, pressing time 14 minutes, resin content $8 \%$ (phenol-formaldehyde), particle moisture content $5 \%$ (dry weight basis). The best results of static bending tests were found when guapuruvu pure and/or in the face layer was used, although the majority of the treatments presented superior values to when to campared the Canadian Standard (CSA O437-0, 1993). In relation to internal band, 50\% of the treatments presented larger values than established by the Canadian Standard. The values of water absorption and thickness swelling were considered high for all the treatments, having to consider that paraffin was not used during board manufacturing.

Key words: particleboard; Oriented Strand Board; Schizolobium parahyba; Erytrina verna.

\section{INTRODUÇÃO}

A chapa de madeira "Oriented Strand Board" (OSB) tem propriedade e uso destinado sobretudo a aplicações estruturais. De acordo com Cloutier (1998), o OSB evoluiu com base em um painel, também estrutural, conhecido como Waferboard que foi introduzido no mercado por volta de 1962.

Como no compensado, as propriedades da madeira são otimizadas quando se produz painéis do tipo OSB, em razão ao posicionamento dos flocos com as fibras em duas direções preferenciais, perpendiculares entre si, conferindo-lhe maior resistência quando comparado com outros painéis de partículas (Einsfeld, 1998).

As vantagens da utilização desse tipo de material em relação à madeira sólida são muitas e residem, especialmente, nos aspectos de rendimento em relação ao volume das toras, diminuição da anisotropia, utilização de madeiras de reflorestamento de rápido crescimento e madeiras de densidade média que, na confecção do painel, confere rigidez suficiente para aplicação estrutural (Mendes e Albuquerque, 2000).

1. Acadêmico(a) do Curso de Engenharia Florestal, Instituto de Florestas, Universidade Federal Rural do Rio de Janeiro, Rodovia BR $465 \mathrm{Km} \mathrm{07,} \mathrm{Campus} \mathrm{Universitário,} \mathrm{CEP} \mathrm{23890-000,} \mathrm{Seropédica} \mathrm{(RJ).}$ grazibaptista@bol.com.br

2. Engenheiro Florestal, Dr., Professor Adjunto do Departamento de Produtos Florestais, Instituto de Florestas, Universidade Federal Rural do Rio de Janeiro, Rodovia BR 465 Km 07, Campus Universitário, CEP 23890-000, Seropédica (RJ).edva@ufrrj.br

Recebido para publicação em 7/4/2003 e aceito em 10/5/2004. 
Determinadas características devem ser consideradas para a escolha da madeira a ser utilizada para fabricação de OSB, como: sua densidade, tipo de anéis de crescimento e conteúdo de umidade, sendo a densidade a mais importante entre elas uma vez que influencia determinadas propriedades mecânicas (Cloutier, 1998).

A quantidade de resíduos gerados pela indústria madeireira no Brasil é bastante elevada. Apesar do volume que representam, esses resíduos têm sido pouco utilizados e, quando isso ocorre, grande parte é destinado para produção de energia. Dentre esses resíduos, as maravalhas de madeira representam uma parcela significativa. As maravalhas apresentam boas características para a produção de chapas de partículas, permitindo a sua utilização isoladamente ou em combinação com outras matérias-primas (Brito, 1995).

A maioria dos processos tecnológicos adotados pelas indústrias de produtos florestais possui rígidas exigências em relação à espécie, tamanho e qualidade da matéria-prima, pois tais processos foram quase que totalmente desenvolvidos em países com grandes reservas de florestas homogêneas (Nakamura e Sobral Filho, 1982).

A disponibilidade de estudos sobre uso de espécies nativas para as finalidades supracitadas é praticamente inexpressiva. A utilização de muitas madeiras tropicais é, às vezes, problemática e pouco conhecida, razão pela qual a qualidade das chapas produzidas pode apresentar variações (Nunes, et al 1999).

A intensa devastação e fragmentação da Mata Atlântica causaram e têm causado preocupações, não só pelo aumento do processo erosivo e, conseqüente, assoreamento do sistema hídrico superficial, mas também por promover a extinção de espécies vegetais e animais, em geral muito pouco conhecidas quanto à classificação botânica e potencialidade de uso (Spolidoro, 2001).

As espécies Schizolobium parahyba e Erythrina Verna apresentam características que poderiam viabilizar a produção de OSB, tais como: rápido crescimento, desenvolvimento em sítios bastante descaracterizados e densidade compatível à exigida para fabricação de tal chapa.

Por outro lado, o desconhecimento sobre os aspectos silviculturais e tecnológicos das referidas espécies são fatores limitantes, verificando-se um verdadeiro descompasso ao se considerar que, atualmente, é incontestável a importância do descobrimento de matérias-primas alternativas.

Atualmente, vastas áreas sob domínio da Mata Atlântica como as do vale do rio Paraíba do Sul, um dos principais eixos econômicos do Brasil, se encontram subutilizadas. Tais áreas são ocupadas em sua maioria por pastagens pouco produtivas, o que favorece em especial a degradação dos recursos edáficos, hídricos e, conseqüentemente, o êxodo rural (Costa, 2002).

Considerando a recente instalação da primeira planta de fabricação de chapas OSB no Brasil (estado do Paraná), a silvicultura com essências florestais nativas da região do Vale do Paraíba do Sul, visando à fabricação desses painéis com espécies adaptadas às condições atuais de tais sítios, como é o caso de Schizolobium parahyba e Erytrina verna, poderia servir como uma alternativa de produção para essa região, com benefícios econômicos, ecológicos e sociais.

O objetivo deste trabalho foi produzir chapas de partículas "strand" e maravalhas partindo das espécies nativas da Mata Atlântica Schizolobium parahyba (Vell.) Blake (guapuruvu) e Erytrina verna Vell. (mulungu) e suas combinações.

\section{MATERIAL E MÉTODOS}

Todos os espécimes de Schizolobium parahyba e Erytrina verna foram obtidos no Município de Barra do Piraí-RJ, região do Médio Vale do Paraíba do Sul. Retiroaram amostras partindo da base da árvore a $25,50,75$ e $100 \%$ da altura comercial para a determinação da densidade básica pelo método da imersão em mercúrio.

As partículas foram geradas em um picador de disco, com as dimensões de $90 \times 20 \times 0,5 \mathrm{~mm}$. Enquanto as maravalhas foram obtidas pelo aplainamento da madeira de guapuruvu, sem passar por nenhum processo de classificação. Todas as partículas foram secas ao conteúdo de umidade de 5\% (base peso seco). 
Utilizaram-se 8\% de resina fenólica (Cascophen HL-2080), aplicada por meio de aspersão através de uma pistola à base de ar comprimido, em um aplicador do tipo tambor rotatório.

Foram produzidos trinta painéis, sendo cinco por tratamento, compostos por três camadas na proporção de 1:2:1. As dimensões nominais dos painéis foram de 450 x 450 x $12 \mathrm{~mm}$ e a densidade nominal de $0,65 \mathrm{~g} / \mathrm{cm}^{3}$.

O delineamento experimental constou de seis tratamentos: 100\% de partículas de mulungu (T1); 100\% de partículas de guapuruvu (T2) ; 50\% de partículas de mulungu na capa e 50\% de partículas de guapuruvu no miolo (T3); 50\% de partículas de guapuruvu na capa e 50\% de partículas de mulungu no miolo (T4); 50\% de partículas de guapuruvu na capa e 50\% de maravalhas de guapuruvu no miolo (T5); 50\% de partículas de mulungu na capa e 50\% de maravalhas de guapuruvu no miolo (T6).

A formação do colchão foi realizada manualmente, procedendo à prensagem em 14 minutos sob temperatura de $180^{\circ} \mathrm{C}$. As chapas foram climatizadas de acordo com a norma AMERICAN SOCIETY FOR TESTING MATERIALS (ASTM D 1037-91), sendo os corpos-de-prova confeccionados para os ensaios de flexão estática (módulo de ruptura e módulo de elasticidade paralelo e perpendicular), ligação interna (LI), inchamento em espessura 2 e 24 horas (IE2 e IE24) e absorção de água 2 e 24 horas (AA2 e AA24), de acordo com a mesma norma.

Os resultados da massa específica (densidade) dos painéis foram submetidos à análise de variância. Para avaliar o efeito dos tratamentos foi realizada a análise de variância para todos os ensaios, seguida pelo teste de Tukey a $95 \%$ de probabilidade, toda vez que a hipótese de nulidade foi rejeitada.

\section{RESULTADOS E DISCUSSÃO}

A análise de variância da densidade dos corpos-de-prova mostrou que não houve diferença estatística significativa entre estas, podendo-se dizer que o processo de deposição das partículas foi homogêneo. Os valores médios da densidade dos painéis por tratamento bem como os respectivos coeficientes de variação para os corpos-de-prova, no sentido paralelo e perpendicular às fibras, estão apresentados na Tabela 1.

TABELA 1: Valores médios da densidade dos painéis por tratamentos e os respectivos coeficientes de variação para os corpos de prova paralelo e perpendicular às fibras.

TABLE 1: Average values of board density per treatment and the respective coefficient of variation for the specimen parallel and perpendicular to the fibers.

\begin{tabular}{cccccc}
\hline Tratamento & DEP // $\left(\mathrm{g} / \mathrm{cm}^{3}\right)$ & CV $(\%)$ & DEP $\left(\mathrm{g} / \mathrm{cm}^{3}\right)$ & CV $(\%)$ \\
\hline T1 & $0,67 \mathrm{a}$ & 8,03 & $0,69 \mathrm{a}$ & 6,11 \\
T2 & $0,67 \mathrm{a}$ & 9,00 & $0,68 \mathrm{a}$ & 7,04 \\
T3 & $0,68 \mathrm{a}$ & 3,41 & $0,70 \mathrm{a}$ & 5,08 \\
T4 & $0,64 \mathrm{a}$ & 2,80 & $0,66 \mathrm{a}$ & 4,33 \\
T5 & $0,64 \mathrm{a}$ & 2,10 & $0,67 \mathrm{a}$ & 6,22 \\
T6 & $0,66 \mathrm{a}$ & 2,91 & $0,64 \mathrm{a}$ & 4,83 \\
\hline
\end{tabular}

Em que: $\mathrm{DEP}=$ densidade dos painéis; $\mathrm{CV}=$ coeficiente de variação; $/ /=$ corpos-de-prova no sentido paralelo; $\perp^{\perp}=$ corpos-de-prova no sentido perpendicular. Médias seguidas de mesma letra não diferem entre si comparadas pelo teste de Tukey ao nível de 5\% de probabilidade de erro.

As densidades básicas de guapuruvu e mulungu foram 0,45 e $0,25 \mathrm{~g} / \mathrm{cm}^{3}$ respectivamente, demonstrando que tais espécies estão na faixa de densidade para a produção de painéis OSB mencionada por Cloutier (1998). Baseado nisso, partiu-se da premissa que painéis OSB produzidos com base nessas espécies poderiam apresentar performances similares aos produzidos pelas indústrias Canadenses e Americanas de OSB, pois segundo Maloney (1993), a densidade é a variável mais importante relativa à espécie que regula as propriedades da chapa. 


\section{Módulo de ruptura (MOR) e Módulo de elasticidade (MOE)}

Na Tabela 2, estão apresentados os valores de MOR e MOE paralelo e perpendicular para todos os tratamentos. Os valores médios de MOR e MOE paralelo dos painéis de todos os tratamentos foram superiores ao sentido perpendicular, corroborando com os resultados de Zhou (1990). Os valores médios de MOR paralelo para todos os tratamentos variaram de 406 a $266 \mathrm{kgf} / \mathrm{cm}^{2}$, enquanto que o MOE variou de 83713 a $48297 \mathrm{kgf} / \mathrm{cm}^{2}$ (Tabela 2). O melhor resultado para MOR paralelo foi o do tratamento T2, apresentando diferença estatística dos tratamentos T1, T5 e T6. Os demais tratamentos não apresentaram diferença significativa entre si.

TABELA 2: Resultados de Flexão estática dos painéis produzidos.

TABLE 2: Results of static bending tests of the panels.

\begin{tabular}{|c|c|c|c|c|c|c|c|c|c|c|c|c|}
\hline \multirow{3}{*}{ Ensaios } & \multicolumn{2}{|c|}{$\mathrm{T} 1$} & \multicolumn{2}{|c|}{$\mathrm{T} 2$} & \multicolumn{2}{|c|}{ T3 } & \multicolumn{2}{|c|}{$\mathrm{T} 4$} & \multicolumn{2}{|c|}{ T5 } & \multicolumn{2}{|c|}{ T6 } \\
\hline & \multicolumn{2}{|c|}{$\left(\mathrm{kgf} / \mathrm{cm}^{2}\right)$} & \multicolumn{2}{|c|}{$\left(\mathrm{kgf} / \mathrm{cm}^{2}\right)$} & \multicolumn{2}{|c|}{$\left(\mathrm{kgf} / \mathrm{cm}^{2}\right)$} & \multicolumn{2}{|c|}{$\left(\mathrm{kgf} / \mathrm{cm}^{2}\right)$} & \multicolumn{2}{|c|}{$\left(\mathrm{kgf} / \mathrm{cm}^{2}\right)$} & \multicolumn{2}{|c|}{$\left(\mathrm{kgf} / \mathrm{cm}^{2}\right)$} \\
\hline & $/ /$ & $\perp$ & $/ /$ & $\perp$ & $/ /$ & $\perp$ & $/ /$ & $\perp$ & $/ /$ & $\perp$ & $/ /$ & $\perp$ \\
\hline MOR & $254 \mathrm{a}$ & $204 c$ & $406 \mathrm{~b}$ & $343 d$ & $319 \mathrm{ab}$ & $160 \mathrm{c}$ & $327 \mathrm{ab}$ & $240 \mathrm{c}$ & $266 \mathrm{a}$ & $227 \mathrm{c}$ & $236 \mathrm{a}$ & $208 \mathrm{c}$ \\
\hline MOE & $55163 \mathrm{e}$ & $30038 \mathrm{~g}$ & $83713 \mathrm{f}$ & $62850 \mathrm{~h}$ & $55334 \mathrm{e}$ & $24563 \mathrm{~g}$ & $65947 \mathrm{ef}$ & $37834 \mathrm{~g}$ & $62130 \mathrm{e}$ & $39797 \mathrm{~g}$ & $48297 \mathrm{e}$ & $29447 \mathrm{~g}$ \\
\hline
\end{tabular}

Médias seguidas de mesma letra não diferem entre si comparadas pelo teste de Tukey ao nível de 5\% de probabilidade de erro.

Os painéis OSB de guapuruvu (T2) apresentaram valor médio de MOR (406 kgf/ $\left.\mathrm{cm}^{2}\right)$ no sentido paralelo, inferiores aos encontrados por Sobral Filho (1979) de $428 \mathrm{kgf} / \mathrm{cm}^{2}$ e dos painéis comerciais americanos $\left(429 \mathrm{kgf} / \mathrm{cm}^{2}\right)$. Os valores médios dos tratamentos T1, T5 e T6 se apresentaram abaixo dos mínimos estabelecidos pela norma supracitada. Pode-se observar que os melhores resultados foram obtidos quando as partículas de guapuruvu foram utilizadas na capa, pois o tratamento $\mathrm{T} 4$ foi maior que o tratamento T3 assim como o tratamento T5 foi superior ao a tratamento T6.

Os resultados de MOR paralelo obtido para os tratamentos T5 e T6 foram inferiores àqueles obtidos para os demais tratamentos e os valores exigidos pela norma CSA 1993, para as chapas O-2. Entretanto, foram superiores aos valores exigidos para chapas do tipo O-1, demonstrando assim a viabilidade de utilização da maravalha no miolo das chapas. A redução nos valores de MOR paralelo observados nos tratamentos T5 e T6, estão de acordo com o observado por Brito (1995), em que chapas produzidas partindo de maravalhas apresentaram resultados inferiores em resistência, dureza e estabilidade dimensional quando comparadas às chapas feitas de flocos. Resultados semelhantes foram encontrados por Lima et al (2002), os quais produziram experimentalmente chapas utilizando partículas strands de Pinus em combinação com maravalha da própria espécie encontrando melhores resultados para MOE perpendicular e paralelo, bem como MOR perpendicular, quando utilizou chapas com $100 \%$ de partículas strand. Porém, para o MOR paralelo, o mesmo autor encontrou resultado superior quando utilizou $50 \%$ de maravalhas.

O melhor tratamento para o MOE paralelo foi o T2, sendo estatisticamente igual ao tratamento T4 e diferente dos demais. O valor médio do MOE paralelo para o T2 $\left(83.713 \mathrm{kgf} / \mathrm{cm}^{2}\right)$ se mostrou acima dos valores dos painéis comerciais americanos de $54.656 \mathrm{kgf} / \mathrm{cm}^{2}$ (Mendes, 2001) e dos encontrados por Sobral Filho (1979), de $66.313 \mathrm{kgf} / \mathrm{cm}^{2}$. Todos os tratamentos, com exceção do T6 foram superiores aos valores mínimos exigidos pela norma CSA (1993), para chapas do tipo O-2. O tratamento T6, foi superior aos valores mínimos exigidos para chapas do tipo O-1. Da mesma forma que o observado para o MOR paralelo, os melhores resultados foram encontrados para os tratamentos nos quais o guapuruvu foi utilizado como capa. A Tabela 3 apresenta as propriedades físicas e mecânicas por categorias de OSB definidas na Norma CSA O437-0.

Os valores médios de MOR perpendicular para todos os tratamentos variaram de 160 a $343 \mathrm{kgf} / \mathrm{cm}^{2}$, enquanto que o MOE variou de 24.563 a $62.850 \mathrm{kgf} / \mathrm{cm}^{2}$ (Tabela 2). Nesse parâmetro, tanto para MOR quanto para MOE, o tratamento que apresentou o melhor resultado foi o T2, diferindo estatisticamente dos demais tratamentos os quais não apresentaram diferença estatística entre si.

O valor médio de MOR perpendicular do T2 $\left(343 \mathrm{kgf} / \mathrm{cm}^{2}\right)$ é superior aos valores mínimos 
estabelecidos pela CSA (1993) de $124 \mathrm{kgf} / \mathrm{cm}^{2}$.

O valor médio de MOE perpendicular $\left(62.850 \mathrm{kgf} / \mathrm{cm}^{2}\right)$ do T2 $(100 \%$ de partículas strand de guapuruvu) foi superior aos encontrados na literatura, inclusive ficando bem acima do valor mínimo estabelecido pela CSA (1993) de $43.000 \mathrm{kgf} / \mathrm{cm}^{2}$. Todos os tratamentos apresentaram valores médios para MOR e MOE perpendicular superiores aos estabelecidos pela norma supracitada, categoria O-1. Mais uma vez, os tratamentos, em que o guapuruvu foi utilizado integralmente ou na capa, apresentaram os melhores resultados.

TABELA 3: Propriedades físicas e mecânicas do OSB definido na Norma CSA O437-0.

TABLE 3: Physical and mechanical properties of OSB defined in CSA O437-0 Standard.

\begin{tabular}{lcc}
\hline Propriedades & $0-2$ & $0-1$ \\
\hline Chapa seca pronta para exportação: & & 234 \\
Módulo de ruptura paralelo $\left(\mathrm{kgf} / \mathrm{cm}^{2}\right)$ & 290 & 96 \\
Módulo de ruptura perpendicular $\left(\mathrm{kgf} / \mathrm{cm}^{2}\right)$ & 124 & 45000 \\
Módulo de elasticidade paralelo $\left(\mathrm{kgf} / \mathrm{cm}^{2}\right)$ & 55000 & 43000 \\
Módulo de elasticidade perpendicular $\left(\mathrm{kgf} / \mathrm{cm}^{2}\right)$ & 15000 & 3,45 \\
Ligação interna $\left(\mathrm{kgf} / \mathrm{cm}^{2}\right)$ & 3,45 & \\
\hline
\end{tabular}

Fonte: Adaptado da CANIDIAN STANDARDS ASSOCIATION (1993).

Os melhores resultados apresentados pelos tratamentos compostos por guapuruvu, integralmente ou na capa, tanto para MOR como MOE, podem ser explicados pelo fato de que o mulungu, por apresentar menor densidade e conseqüentemente área superficial específica das partículas maior que aquelas apresentadas pelas partículas de guapuruvu, proporcionou uma maior dispersão do adesivo e, conseqüentemente, uma menor adesão entre as partículas.

\section{Absorção de água (AA)}

Na Tabela 4, são apresentados os valores médios da absorção de água (2 e 24h) dos tratamentos. A menor AA $2 \mathrm{~h}$ e AA $24 \mathrm{~h}$ foi apresentada pelo tratamento T2, que foi diferente estatisticamente apenas do tratamento T6. Conforme dito no item acima, os maiores valores encontrados para AA $2 \mathrm{~h}$ e $24 \mathrm{~h}$ para o tratamento T6 poderiam ser justificado em razão do uso de mararvalhas na composição das chapas uma vez que esse tipo de partículas influencia a estabilidade dimensional das chapas.

Os valores médios da AA para o T2 a $2 \mathrm{~h}$ e $24 \mathrm{~h}$ foram, respectivamente, $21 \%$ e $45 \%$, sendo inferiores aos encontrados por Mendes (2001) 37,17\% (2h) e 63,75\% (24h) para painéis OSB de espécies de pinus com densidade nominal de $0,65 \mathrm{~g} / \mathrm{cm}^{3}$.

TABELA 4: Média dos valores para absorção de água - AA (\%) de todos os tratamentos.

TABLE 4: Average values of water absorption (\%) for all the treatments.

\begin{tabular}{|c|c|c|c|c|c|c|c|c|c|c|c|c|}
\hline \multirow{2}{*}{ Ensaio } & \multicolumn{2}{|c|}{$\mathrm{T} 1$} & \multicolumn{2}{|c|}{$\mathrm{T} 2$} & \multicolumn{2}{|c|}{ T3 } & \multicolumn{2}{|c|}{$\mathrm{T} 4$} & \multicolumn{2}{|c|}{ T5 } & \multicolumn{2}{|c|}{ T6 } \\
\hline & \multicolumn{2}{|c|}{$(\%)$} & \multicolumn{2}{|c|}{$(\%)$} & \multicolumn{2}{|c|}{$(\%)$} & \multicolumn{2}{|c|}{$(\%)$} & \multicolumn{2}{|c|}{$(\%)$} & \multicolumn{2}{|c|}{$(\%)$} \\
\hline \multirow{2}{*}{ AA } & $2 \mathrm{~h}$ & $24 \mathrm{~h}$ & $2 \mathrm{~h}$ & $24 \mathrm{~h}$ & $2 \mathrm{~h}$ & $24 \mathrm{~h}$ & $2 \mathrm{~h}$ & $24 \mathrm{~h}$ & $2 \mathrm{~h}$ & $24 \mathrm{~h}$ & $2 \mathrm{~h}$ & $24 \mathrm{~h}$ \\
\hline & $29 \mathrm{ab}$ & $50 \mathrm{~cd}$ & $21 \mathrm{a}$ & $45 c$ & $28 \mathrm{ab}$ & $51 \mathrm{~cd}$ & $26 a b$ & $53 \mathrm{~cd}$ & $30 \mathrm{ab}$ & $50 \mathrm{~cd}$ & $33 b$ & $58 \mathrm{~d}$ \\
\hline
\end{tabular}

Médias seguidas de mesma letra não diferem entre si comparadas pelo teste de Tukey ao nível de 5\% de probabilidade de erro.

Apesar do tratamento T2 ter apresentado diferença estatística apenas em relação ao tratamento T6, pode-se observar que os menores valores de inchamento foram encontrados para os tratamentos em que o guapuruvu foi utilizado integralmente ou na capa.

Mendes (2001) sugere que os altos valores para a absorção de água encontrado em seu estudo podem ser pelo ao fato de não ter sido utilizada emulsão de parafina na manufatura dos painéis. A mesma tendência foi encontrada para os painéis de todos os tratamentos deste estudo, considerando que estes também não receberam emulsão de parafina na manufatura das chapas. 


\section{Inchamento em espessura (IE)}

$\mathrm{Na}$ Tabela 5, são apresentados os valores médios do inchamento em espessura (2 e 24h) dos tratamentos. Segundo Brito (1995), o inchamento em espessura é uma das propriedades mais importantes em termos de estabilidade dimensional dos painéis, e pode ser afetada pela espécie de madeira, geometria das partículas, nível de resina, eficiência da aplicação da cola e condições de prensagem.

TABELA 5: Média dos valores para inchamento em espessura - IE (\%) de todos os tratamentos.

TABLE 5: Average values of thickness swelling (\%) for all treatments.

\begin{tabular}{|c|c|c|c|c|c|c|c|c|c|c|c|c|}
\hline \multirow{2}{*}{ Ensaio } & \multicolumn{2}{|c|}{$\mathrm{T} 1$} & \multicolumn{2}{|c|}{$\mathrm{T} 2$} & \multicolumn{2}{|c|}{ T3 } & \multicolumn{2}{|c|}{$\mathrm{T} 4$} & \multicolumn{2}{|c|}{ T5 } & \multicolumn{2}{|c|}{ T6 } \\
\hline & \multicolumn{2}{|c|}{$(\%)$} & \multicolumn{2}{|c|}{$(\%)$} & \multicolumn{2}{|c|}{$(\%)$} & \multicolumn{2}{|c|}{$(\%)$} & \multicolumn{2}{|c|}{$(\%)$} & \multicolumn{2}{|c|}{$(\%)$} \\
\hline \multirow{2}{*}{ IE } & $2 \mathrm{H}$ & $24 \mathrm{~h}$ & $2 \mathrm{~h}$ & $24 \mathrm{~h}$ & $2 \mathrm{~h}$ & $24 \mathrm{~h}$ & $2 \mathrm{~h}$ & $24 \mathrm{~h}$ & $2 \mathrm{~h}$ & $24 \mathrm{~h}$ & $2 \mathrm{~h}$ & $24 \mathrm{~h}$ \\
\hline & $33 \mathrm{ab}$ & $46 \mathrm{~cd}$ & $25 \mathrm{a}$ & $48 \mathrm{c}$ & $41 b$ & $61 \mathrm{~d}$ & $31 \mathrm{ab}$ & $53 \mathrm{~cd}$ & $36 \mathrm{ab}$ & $53 \mathrm{~cd}$ & $24 a$ & $41 \mathrm{c}$ \\
\hline
\end{tabular}

Médias seguidas de mesma letra não diferem entre si comparadas pelo teste de Tukey ao nível de $5 \%$ de probabilidade de erro.

$\mathrm{O}$ menor IE $2 \mathrm{~h}$ e $24 \mathrm{~h}$ foi obtido para o tratamento $\mathrm{T} 6$, que diferiu significativamente apenas do tratamento T3. Os valores médios do IE para os painéis T6 a $2 \mathrm{~h}$ e $24 \mathrm{~h}$ foram, respectivamente, 24 e $41 \%$, sendo inferior ao encontrado por Mendes (2001) para 2h (26\%) e superior ao de 24h (34\%), para painéis OSB de várias espécies de pinus com densidade nominal de $0,65 \mathrm{~g} / \mathrm{cm}^{3}$. Entretanto, todos os tratamentos apresentaram resultados acima do estabelecido pela norma da CANADIAN STANDARDS ASSOCIATION (1993).

Wang e Winistorfer (2000) observaram que painéis OSB produzidos com espécie de menor densidade apresentaram maior inchamento em espessura quando comparados com espécies de maior densidade, resultando em uma maior razão de compactação. Neste trabalho, os resultados não apresentaram a mesma tendência observada pelos autores anteriores, pois o tratamento $\mathrm{T} 1$ apresentou valores inferiores ao tratamento T2.

Segundo Mendes (2000), a maioria das citações relata a existência de uma relação positiva entre a densidade dos painéis e as propriedades de IE e AA, o que poderia então justificar o maior valor encontrado para inchamento em espessura para o tratamento T3 uma vez que este apresenta a maior densidade dos painéis.

\section{Ligação interna (LI)}

Na Tabela 6, são apresentados os valores médios da ligação interna dos tratamentos. O tratamento T1 apresentou o melhor resultado para a LI, embora não tenha apresentado diferença significativa dos tratamentos $\mathrm{T} 2$, T4 e T6.

TABELA 6: Média dos valores para ligação interna - LI $\left(\mathrm{kgf} / \mathrm{cm}^{2}\right)$ de todos os tratamentos.

TABLE 6: Average values for internal band $\left(\mathrm{kgf} / \mathrm{cm}^{2}\right)$ for all treatments.

\begin{tabular}{c|c|c|c|c|c|c}
\hline \multirow{2}{*}{ Ensaio } & $\mathrm{T} 1$ & $\mathrm{~T} 2$ & $\mathrm{~T} 3$ & $\mathrm{~T} 4$ & $\mathrm{~T} 5$ & $\mathrm{~T} 6$ \\
\cline { 2 - 7 } & $\left(\mathrm{kgf} / \mathrm{cm}^{2}\right)$ & $\left(\mathrm{kgf} / \mathrm{cm}^{2}\right)$ & $\left(\mathrm{kgf} / \mathrm{cm}^{2}\right)$ & $\left(\mathrm{kgf} / \mathrm{cm}^{2}\right)$ & $\left(\mathrm{kgf} / \mathrm{cm}^{2}\right)$ & $\left(\mathrm{kgf} / \mathrm{cm}^{2}\right)$ \\
\hline LI & $4,25 \mathrm{a}$ & $3,87 \mathrm{ab}$ & $3,25 \mathrm{~b}$ & $3,92 \mathrm{ab}$ & $3,36 \mathrm{~b}$ & $3,44 \mathrm{ab}$
\end{tabular}

Médias seguidas de mesma letra não diferem entre si comparadas pelo teste de Tukey ao nível de 5\% de probabilidade de erro.

O valor médio da ligação interna do T1 $\left(4,25 \mathrm{kgf} / \mathrm{cm}^{2}\right)$ foi inferior ao encontrado por Mendes (2001) de $6,41 \mathrm{kgf} / \mathrm{cm}^{2}$ para chapas de mesma densidade nominal $\left(0,65 \mathrm{~g} / \mathrm{cm}^{3}\right)$. No entanto, foi superior ao valor mínimo estabelecido pela norma canadense CSA O437-0 (1993) de 3,45 kgf $/ \mathrm{cm}^{2}$. Os tratamentos T3, T5 e T6 apresentaram valores médios inferiores ao estabelecido pela mesma norma.

Em geral, a literatura menciona que com o aumento da densidade dos painéis e outras variáveis, ocorre um aumento na resistência a ligação interna. Apesar do tratamento T3 ter apresentado a maior 
densidade dos painéis, o mesmo tratamento não apresentou o melhor resultado para LI.

Segundo Brito (1995), os bordos em formas de pena nas partículas de maravalha proporcionam alta resistência à ligação interna. Porém, as chapas produzidas com esse tipo de partícula apresentaram os menores valores para LI demonstrando que a mistura de espécies e partículas, nesse caso, não foi satisfatória. Já Lima et al (2002) encontraram o melhor resultado para LI quando utilizaram 100\% de partículas maravalhas.

Mendes (2000) relata que alguns extrativos da madeira podem causar estouro e que espécies com baixo teor de extrativos são mais desejáveis para a produção de OSB. O pH da madeira também é considerado outra variável importante na produção de painéis OSB. Vale ressaltar que as duas variáveis supracitadas são desconhecidas para as espécies estudadas nesta pesquisa e que tais variáveis poderiam explicar melhor os resultados encontrados para LI.

A ligação interna e o tempo de prensagem utilizados na manufatura dos painéis de OSB têm estreita relação com a espécie utilizada e o teor de umidade das partículas. De acordo com Cloutier (1998), elevados teores de umidade das camadas superficiais do colchão de OSB, em geral, diminuem a densidade da camada interna elevando os riscos de estouro, além de reduzir a ligação interna. Por outro lado, Matos (1988) relata que reduções no tempo de prensagem dos painéis são um tanto desejáveis, implicando em maior produtividade e menor consumo de energia. Segundo Maloney (1993), algumas espécies requerem mais que outras um controle da umidade mais preciso das partículas, em decorrência da possibilidade de ocorrência de estouro ou separação de camadas durante a secagem final dos painéis.

Os painéis de OSB produzidos neste trabalho, enquadram-se, a princípio, no contexto acima por duas razões principais. A primeira concerne ao elevado tempo de prensagem utilizado (14 minutos), em razão da ocorrência de estouro em tempos menores em relação aos satisfatórios 8 minutos utilizados experimentalmente por Iwakiri (1989). Tal problema, pode ter ocorrido em consequência de características inerentes às madeiras de Schizolobium parahyba e Erytrina verna que as tornam propensas ao estouro considerando-se a umidade que foi utilizada para a formação do colchão. A segunda razão se refere ao baixo valor de LI, que segundo Cloutier (1998) pode ser por causa de altos teores de umidade nas camadas superficiais do colchão.

Portanto, torna-se necessária a realização de experimentos envolvendo outros teores de umidade com o intuito de melhorar as propriedades físico-mecânicas de painéis OSB produzidos com madeira de Schizolobium parahyba, Erytrina verna e suas combinações, considerando a elevada potencialidade destas espécies demonstrada nesta pesquisa.

\section{CONCLUSÕES}

Os melhores resultados para o ensaio de flexão estática foram encontrados quando se utilizou guapuruvu puro e/ou na capa, embora a maioria dos tratamentos tenha apresentado valores superiores aos estabelecidos pela norma canadense (CSA O437-0, 1993);

Em relação à ligação interna, 50\% dos tratamentos apresentaram valores superiores aos estabelecidos pela norma canadense, dentre os quais estão presentes as duas espécies de madeira estudadas;

Quanto às propriedades de inchamento em espessura e absorção de água, todos os tratamentos apresentaram valores elevados, devendo-se considerar que não foi utilizado parafina para a confecção das chapas;

Existe a necessidade de estudos relacionados ao $\mathrm{pH}$ da madeira bem como os extrativos presentes na madeira de guapuruvu e mulungu;

As espécies Schizolobium parahyba (Vell.) Blake (guapuruvu) e Erytrina verna Vell. (mulungu), puras ou em misturas, apresentaram elevado potencial para a produção de painéis OSB.

\section{REFERÊNCIAS BIBLIOGRÁFICAS}

AMERICAN SOCIETY FOR TESTING MATERIALS. Standart methods of evaluating the properties of woodbase fiber particle materials. Philadelphia, 1991. v.04-09 (Annual Book of ASTM standarts).

BRITO, E.O. Produção de chapas de partículas de madeira a partir de maravalhas de Pinus elliotti plantado no 
sul do Brasil. 1995. 123p. Tese (Doutorado em Engenharia Florestal)- Universidade Federal do Paraná, Curitiba, 1995.

CANADIAN STANDARDS ASSOCIATION. OSB and Waferboard: CSA 0437.0-93. Ontario, 1993. 18p.

CLOUTIER, A. Oriented StrandBoard (OSB): Raw material, manufacturing process, properties, and uses. In: SEMINÁRIO INTERNACIONAL SOBRE PRODUTOS SÓLIDOS DE MADEIRA DE ALTA TECNOLOGIA 1., E ENCONTRO SOBRE TECNOLOGIA APROPRIADAS DE DESDOBRO, SECAGEM E UTILIZAÇÃO DA MADEIRA DE EUCALIPTO,1.,1998, Belo Horizonte. Anais... Belo Horizonte, 1998. p. 173-185.

COSTA, M.C.R. Avaliação da contaminação ambiental por metais pesados em áreas rurais próximas a uma indústria de reciclagem de chumbo no vale do rio Paraíba do Sul - SP. 2002.195 p Tese (Doutorado em Ciência do Solo)- Universidade Federal Rural do Rio de Janeiro, Rio de Janeiro,2002.

EINSFELD, R. A. et al. Manufatura e características de chapas OSB. In: ENCONTRO BRASILEIRO DE MADEIRAS E ESTRUTURAS DE MADEIRA, 1998, Florianópolis. Anais ...Florianopolis, 1998. v.3, p.385-393.

IWAKIRI, S. Painéis de madeira: n1. Curitiba: FUPEF, 1989. 128p.

LIMA, A.M,; LIMA, R. M.; BRITO, E. O. Produção de aglomerados de partículas "strand" e maravalhas e suas combinações. In: CONGRESSO IBERO-AMERICANO DE PESQUISA E DESENVOLVIMENTO DE PRODUTOS FLORESTAIS, 2., E SEMINARIO EM TECNOLOGIA DA MADEIRA E PRODUTOS FLORESTAIS NÃOMADEIRAVEIS,1., 2002, Curitiba. Anais... Curitiba, 2002.

MATOS, J.L.M. Ciclo de prensa em chapas de partículas estruturais "Waferboard". 1988. 163p. Dissertação (Mestrado em Engenharia Florestal)- Universidade Federal do Paraná, Curitiba, 1988.

MALONEY, T. M. Modern particleboard e dry-process fiberboard manufacturing. 2.ed. São Francisco: M. Freeman, 1993. 689p.

MENDES, L.M. Pinus spp na produção de painéis de partículas orientadas (OSB). 2001. 156p. Tese (Doutorado em Engenharia Florestal)- Universidade Federal do Paraná, Curitiba, 2001.

MENDES, L.M.; ALBUQUERQUE, C.E.C de. Aspectos técnicos e econômicos da indústria brasileira de chapas de fibras e partículas. Revista da Madeira, n.53, p.14-22, 2000.

NAKAMURA, R.M.; SOBRAL FILHO, M. Aglomerado de misturas de espécies tropicais da Amazônia. Brasilia: Ministério da Agricultura, Instituto Brasileiro de Desenvolvimento Florestal, Departamento de Economia Florestal, Laboratório de Produtos Florestais, 1982.

NUNES, W.H.; ANDRADE, A.M. de; BRITO, E.O. Produção de chapas de partículas do estipe de Euterpe edulis Martius (Palmiteiro). Floresta e Ambiente, Seropédica, v.6, n.1, p.95-105, 1999.

SPOLIDORO, M.L.C.V. Composição e estrutura de um trecho de floresta no médio Paraíba do Sul, RJ. 2001. 90p. Dissertação (Mestrado em Ciências Ambientais e Florestais)- Universidade Federal Rural do Rio de Janeiro, Rio de Janeiro, 2001.

SOBRAL FILHO, M. The influence of wood furnish type on properties of oriented strand panels. 1979. 93p. Tese (Doctor Philosophy-major in Forestry)- University of Idaho Graduate School, Idaho, 1979.

WANG, S.; WINISTORFER, P. M. The effect of species and species distribution on the layer characteristics of OSB. Forest Products Journal, Madison, v. 50, n. 4, p. 37-44, 2000.

ZHOU, D. A study of oriented strutural board made from hybrid poplar. Physical and mechanical properties of OSB. Holz Als Roh Und Werkstoff, Berlim, v.48, n.7-8, p.293-296, 1990. 